Check for updates

Cite this: RSC Adv., 2019, 9, 19095

\title{
The potential role of circRNA_004229 in hair/ epidermal regulation after MED1 ablation in keratinocytes $\uparrow$
}

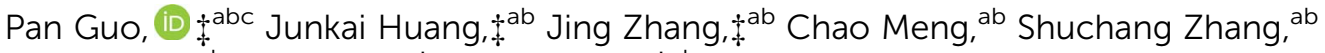 \\ Yunfeng Bai, ${ }^{d}$ Zhiwei Ning ${ }^{\star e}$ and Lizhi Hu ${ }^{\star a b}$
}

Background/Aims: Mediator complex subunit 1 (MED1) is an important transcriptional co-activator involved in multiple signaling pathways. Previous studies indicated an essential role of MED1 in hair cycling and wound repair through regulating the transcription of mRNAs. Circular RNAs (circRNAs), as a novel class of non-coding RNAs, are involved in various skin biological functions. Our study aimed to investigate the circRNAs expression profile in MED1 epidermal conditional knockout mice (KO), and provide potential candidates as well as the mechanism underlying the circRNAs regulation in both hair follicles and epidermis. Method: Microarray based circRNA expression was determined in MED1 KO mice and wild type mice (WT). The expression level was further confirmed by qRT-PCR. We predicted a possible interaction network of circRNA/microRNA/mRNA by bioinformatics and constructed them with Cytoscape software. Expression of several candidate target mRNAs was verified using qRT-PCR. A TUNEL assay was performed to assess the apoptosis level of MED1 KO and WT skin. Results: Here we identified 109 (34-up, 75-down) distinct circRNAs through microarrays that are differently expressed in MED1 KO mice compared with WT mice (FC $>2$ and $p$-value $<0.05)$, suggesting a potential role of circRNAs in epidermal regulation. Among these circRNAs, circRNA_004229 was found to decrease significantly after MED1 deletion. The most likely potential targets miRNA for circRNA_004229 include miR-149-5p and miR-207, which possibly further impede the expression of their target mRNA, Tnfrsf19 and Perp, respectively. Apoptosis was suppressed in MED1 KO mice, which implies a potential role of circRNAs in regulating epidermal biological processes including apoptosis. Conclusion: Our study determined the expression profile of circRNAs in MED1 KO skin, and provided hints that circRNA_004229 might be involved in the regulation of keratinocytes in both hair follicles and interfollicular epidermis through a ceRNA mechanism.

rsc.li/rsc-advances

\section{Introduction}

Mediator complex subunit 1 (MED1), also known as PBP, DRIP205 and TRAP220, is a critical subunit of transcription coactivator Mediator (MED). It has been confirmed that MED1

\footnotetext{
${ }^{a}$ Immunology Department, Key Laboratory of Immune Microenvironment and Disease (Ministry of Education), Tianjin Medical University, Tianjin, 300070, China. E-mail: lizhihu@tmu.edu.cn

${ }^{b}$ Department of Pathogen Biology, School of Basic Medical Sciences, Tianjin Medical University, Tianjin, 300070, China

${ }^{c}$ School of Biomedical Engineering and Technology, Tianjin Medical University, Tianjin, 300070, China

${ }^{a}$ Center of Integrative Medicine, 302 Hospital of Chinese People's Liberation Army, Beijing, 100039, China

${ }^{e}$ Department of Endocrinology and Metabolism, Beijing Chaoyang Hospital, Capital Medical University, Beijing, 100020, China. E-mail: ningzhiwei@hotmail.com

$\dagger$ Electronic supplementary information (ESI) available. See DOI: 10.1039/c9ra02322d

$\ddagger$ These authors contributed equally to this work.
}

actively interacts with a number of nuclear hormone receptors and facilitates their transcription process, including VDR (vitamin D receptor), AR (androgen receptor), TR (thyroid hormone receptor), and PPAR (peroxisome proliferatoractivated receptor) $\cdot^{1-4}$ Previously, we generated a MED1 epidermal conditional knockout (KO) mouse model and investigated its specific function in hair/epidermal regulation. MED1 deletion resulted in aberrant hair follicle (HF) differentiation and cycling defects by affecting Wnt/b-catenin and SHH signaling. ${ }^{5}$ Moreover, hyperproliferation of interfollicular epidermis (IFE) and accelerated wound repair were also observed in MED1 KO mice. Thus, MED1 plays an essential role in regulating the proliferation and differentiation of both IFE and HF. ${ }^{5,6}$

However, considering the abnormal phenotype and complicated responses to different stimulation after MED1 deletion, we anticipate a more intricate mechanism involved in its regulation in epidermis, not confined to proliferation and differentiation. Our precursory research compared the mRNA 
expression profile in MED1 KO mice and WT under unaffected (natural) and wound condition, but left other small RNAs uninvestigated. Recent studies provided strong evidence that endogenous competition mechanism of circular RNAs (circRNAs), microRNA (miRNAs) or long non-coding RNAs (lncRNAs) could influence multiple biological function. ${ }^{7,8}$ Thus, exploring the interaction of these small RNAs after MED1 deletion might enlighten us the role of MED1 in regulation of keratinocytes in a novel way.

CircRNAs are a small group of non-coding RNAs with a closed covalent loop other than linear structure, which are widely distributed in various tissues among eukaryotes. ${ }^{9}$ Because of their high stability and tissue-specific expression, circRNAs are increasingly recognized as relative stable biomarkers for many cancers and other disease. ${ }^{\mathbf{1 0}}$ It was confirmed that circRNAs could regulate expression of abundant target genes by serving as miRNA's 'sponges' and further regulate mRNAs level. ${ }^{\mathbf{1 1 - 1 4}}$ The regulation mechanisms from circRNA to miRNA to genes were shown as Fig. 1. Recent research studied the circRNAs expression profile in different skin diseases. ${ }^{15-17}$ And several circRNAs was proved to participate in the regulation of proliferation, differentiation, and apoptosis of keratinocytes, suggesting that circRNAs might play a role in cutaneous biological process. ${ }^{18,19}$

In this study, we reported the circRNAs expression profile from epidermal samples in MED1 KO mice and wild type mice (WT). CircRNA_004229 was found to decrease significantly after MED1 deletion. CircRNA_004229, also named as mmu_circ_004229 or mmu_circ_0001055 in circBase database (http:// www.circbase.org/), is present in exon1 of Fmn1 (Fig. 1). We further explored the potential involvement of MED1 in regulating hair/epidermal function through circRNA_004229.

\section{Materials and methods}

\section{Mice}

MED1 KO mice were generated by mating floxed MED1 mice (C57/BL6 background) with transgenic mice expressing Cre

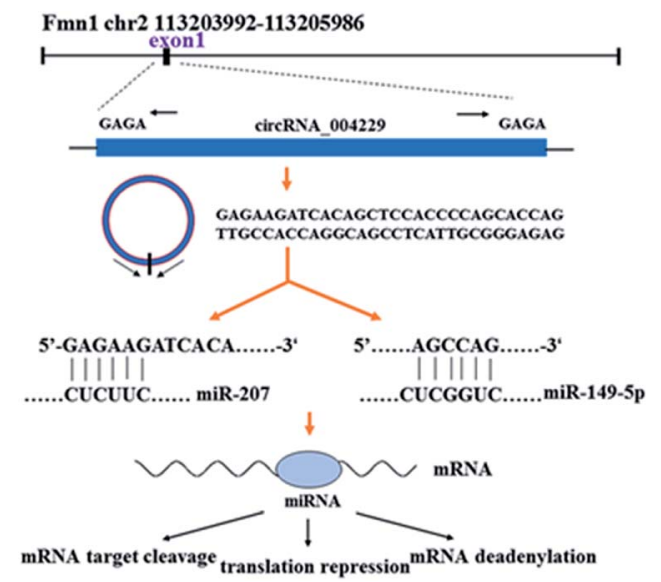

Fig. 1 The simple explanation of circRNA_004229 and its regulation mechanisms from circRNA to miRNA to mRNA. recombinase under the control of keratin 14 (Krt14) promoter (C57/BL6 background). ${ }^{5}$ MED1 was selectively deleted in the keratinocytes of MED1 KO mice, and the genotype of MED1 KO mice and the WT mice was further confirmed by PCR (ESI Fig. $1 \dagger$ ).

All the animals were housed in a virus- and parasite-free animal facility with temperature of $22 \pm 1{ }^{\circ} \mathrm{C}$, relative humidity of $50 \pm 1 \%$ and a light/dark cycle of $12 / 12 \mathrm{~h}$. All animals had free access to soft chow and drinking water. Furthermore, all animal procedures were performed in accordance with the Guidelines for Care and Use of Laboratory Animals and were approved by the Animal Ethics Committee of Tianjin Medical University.

\section{RNA isolation}

Total RNA was isolated from MED1 KO mice and WT mice using the Trizol reagent (Invitrogen, 15596-018, USA). RNA quantification and quality were determined by NanoDrop ND-1000 instrument (Thermo, USA). RNA Integrity and gDNA contamination were tested by Denaturing Agarose Gel Electrophoresis.

\section{Microarray analysis}

The sample preparation and microarray hybridization were performed based on the standard protocols of Arraystar Inc (Rockville, MD, USA). Briefly, total RNAs were digested with RNase R (Epicentre, Inc., USA) to remove linear RNAs and enrich circRNAs. Then, the enriched circRNAs were amplified and transcribed into fluorescent cRNA utilizing a random priming method (Arraystar Super RNA Labeling Kit; Arraystar Inc). Subsequently, the cRNAs were hybridized using Arraystar Mouse circRNA Array $(8 \times 15 \mathrm{~K}$, Arraystar Inc). Washed the slides and then scanned by the Agilent Scanner G2505C (Jamul, CA, USA). Subsequent data processing was performed using the $\mathrm{R}$ software limma package. Foldchanges of $>2.0$ and $P$-values of $<0.05$ were regarded as significantly differentially expressed.

\section{CeRNA analysis and target prediction}

We constructed mmu_circRNA_004229-miRNA-target genes networks using Cytoscape software 3.5.0 to visualize their interactions based on our circRNAs microarray data and the qRT-PCR results for circRNAs and mRNAs. We predicted the circRNA/miRNA interaction using Arraystar's home-made miRNA target prediction software and listed the top 5 putative miRNAs of differential expressed circRNAs. The target genes of specific miRNA were identified with TargetScan, miRPathDB, and miRDB.

\section{Quantitative real-time PCR for circRNAs and mRNAs}

Total RNA was reversely transcribed into cDNA using SuperScriptTM III Reverse Transcriptase (Invitrogen) for circRNAs and reversely transcribed into cDNA using RevertAid First Strand cDNA Synthesis Kit (Thermo Scientific, K1622, USA) for Tnfrsf19 and Perp mRNA. The procedure for preparation 

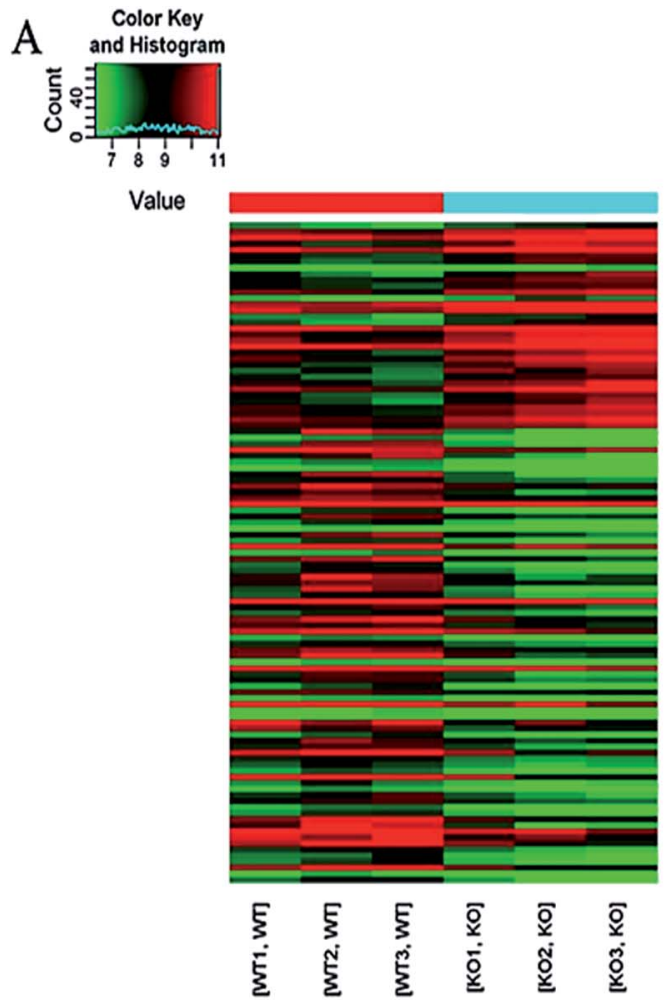
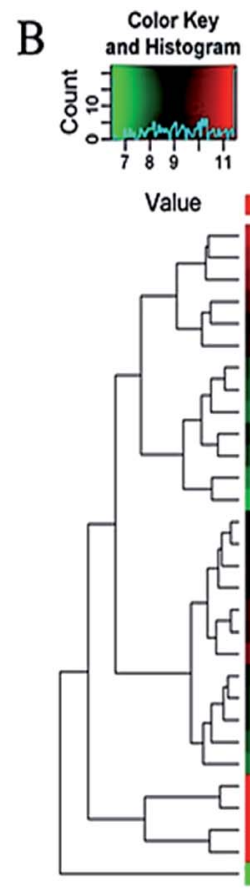
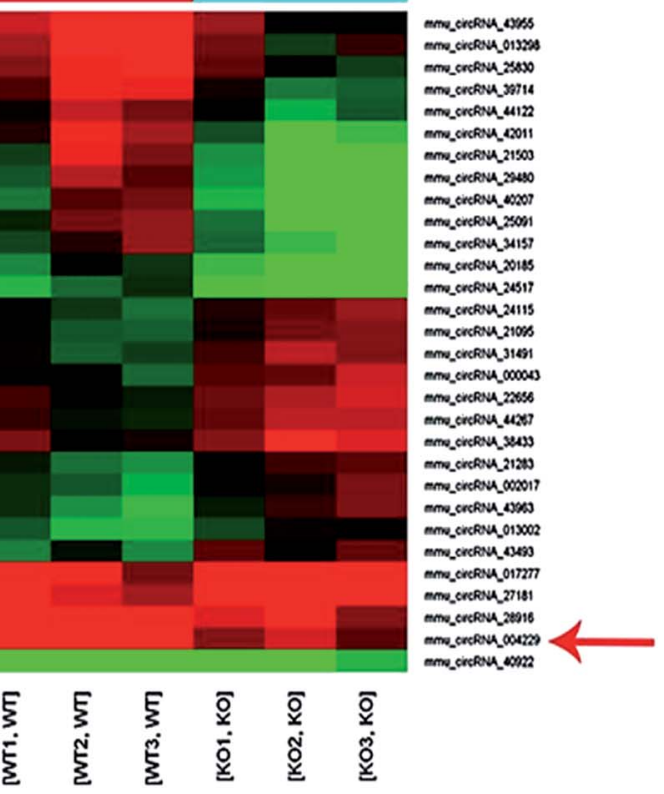

Fig. 2 Heat maps of the differentially expressed circRNAs in dorsal epidermis from MED1 KO mice compared with WT mice. (A) The cluster heat map showed all differentially expressed circRNAs in MED1 KO mice vs. WT mice (FC $>2.0$ and $p$-value $<0.05)$. (B) The hierarchical clustering of the top 15 up- and down-regulated circRNAs in MED1 KO mice vs. WT mice. Rows represent circRNAs, while columns represent compared groups. The expression index is colored in red indicating high expression level, and in green indicating low expression level. The red arrow indicates circRNA_004229.

of cDNA was standard. Quantification of mmu_circRNA_004229 was performed using ViiA 7 Real-time PCR System (Applied Biosystems). Quantification of Tnfrsf19 and Perp mRNA were performed using 7500 Fast Real-Time PCR System (Applied Biosystems). Relative circRNA and mRNAs levels of each sample were determined by the $\Delta \Delta C_{\mathrm{t}}$ method with GAPDH. Q-PCR Primers corresponding to mmucircRNA_004229, Perp, Tnfrsf19 and GAPDH sequences were as follows: for mmu_circRNA_0042229 (sense: $5^{\prime}$ CTCCACCCCAGCACCAGTTG-3', antisense: 5' ${ }^{\prime}$-TGACAGTCCTCTCCCGCAATG-3'); for Tnfrsf19 (sense: $5^{\prime}$ TTCTGTGGGGGACACGATG-3', antisense: 5'-AGAAAATTCAGCGCAGATGGAA-3'); for Perp (sense: $5^{\prime}$-AGACCTTCAGGCTTCACGAT- ${ }^{\prime}$, antisense: $5^{\prime}$ AGGTCATCCTCGTAGTTGGG-3') for GAPDH (sense: $5^{\prime}$ CACTGAGCAAGAGAGGCCCTAT- $3^{\prime}$ antisense: 5'-GCAGCGAACTTTATTGATGGTATT-3').

\section{TUNEL assay}

Full-thickness skin tissue from the back of age-matched WT and MED1 KO mice were fixed overnight in 4\% paraformaldehyde. Then the tissue was dehydrated, and embedded in paraffin using standard techniques. Consecutive $5 \mu \mathrm{m}$ serial sections were deparaffinized, rehydrated, and washed with $1 \times$ phosphate-buffered saline solution (PBS) for three times. After that, slides were incubated in permeabilization solution for 30 min and rinsed with $1 \times$ PBS for three times. Then the slides were incubated with blocking solution for $10 \mathrm{~min}$ at $15-25{ }^{\circ} \mathrm{C}$ and washed with $1 \times$ PBS for three times. The total volume $(5 \mu \mathrm{L})$ of enzyme solution was added to the remaining $45 \mu \mathrm{L}$ label solution to obtain $50 \mu \mathrm{L}$ TUNEL (KeyGEN BioTECH, Jiangsu, China) mixture for each slide, and mixed well to equilibrate components. Slides were incubated with it for $1 \mathrm{~h}$ at $37{ }^{\circ} \mathrm{C}$. Finally, they were stained with Hematoxylin (Solarbio G1140, Beijing, China) for $2 \mathrm{~min}$ followed by examination under a microscope (OLYMPUS). TUNEL ${ }^{+}$cells were counted by selecting 3 fields with the most TUNEL ${ }^{+}$cells. For each field, all TUNEL $^{+}$and TUNEL ${ }^{-}$cells were counted. Percent of TUNEL ${ }^{+}$ cells were calculated as ((total $\mathrm{TUNEL}^{+}$cells $) /($total cells $) \times$ $100 \%)$.

\section{Immunohistochemistry}

$5 \mu \mathrm{m}$ serial skin sections were adhered to poly-L-lysine-coated slides, and deparaffinized in xylene and rehydrated with alcohol solutions. Briefly, high-temperature antigen retrieval was achieved by heating the samples in $0.01 \mathrm{M}$ citrate buffer at $95{ }^{\circ} \mathrm{C}$ for $10 \mathrm{~min}$, then the samples were immersed into methanol containing $0.3 \% \mathrm{H}_{2} \mathrm{O}_{2}$ to inactivate endogenous peroxidase at room temperature for $30 \mathrm{~min}$. The nonspecific background was blocked using $1 \%$ BSA and $5 \%$ goat serum in 
A

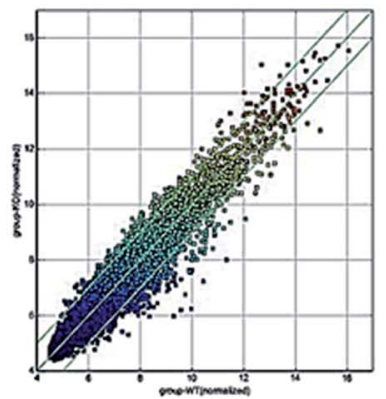

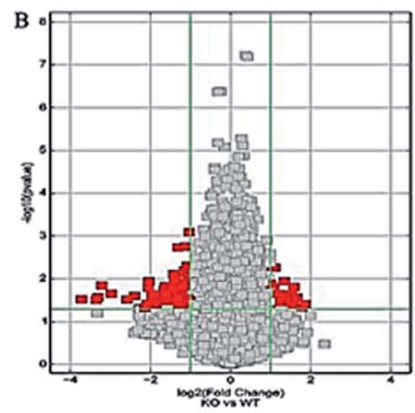
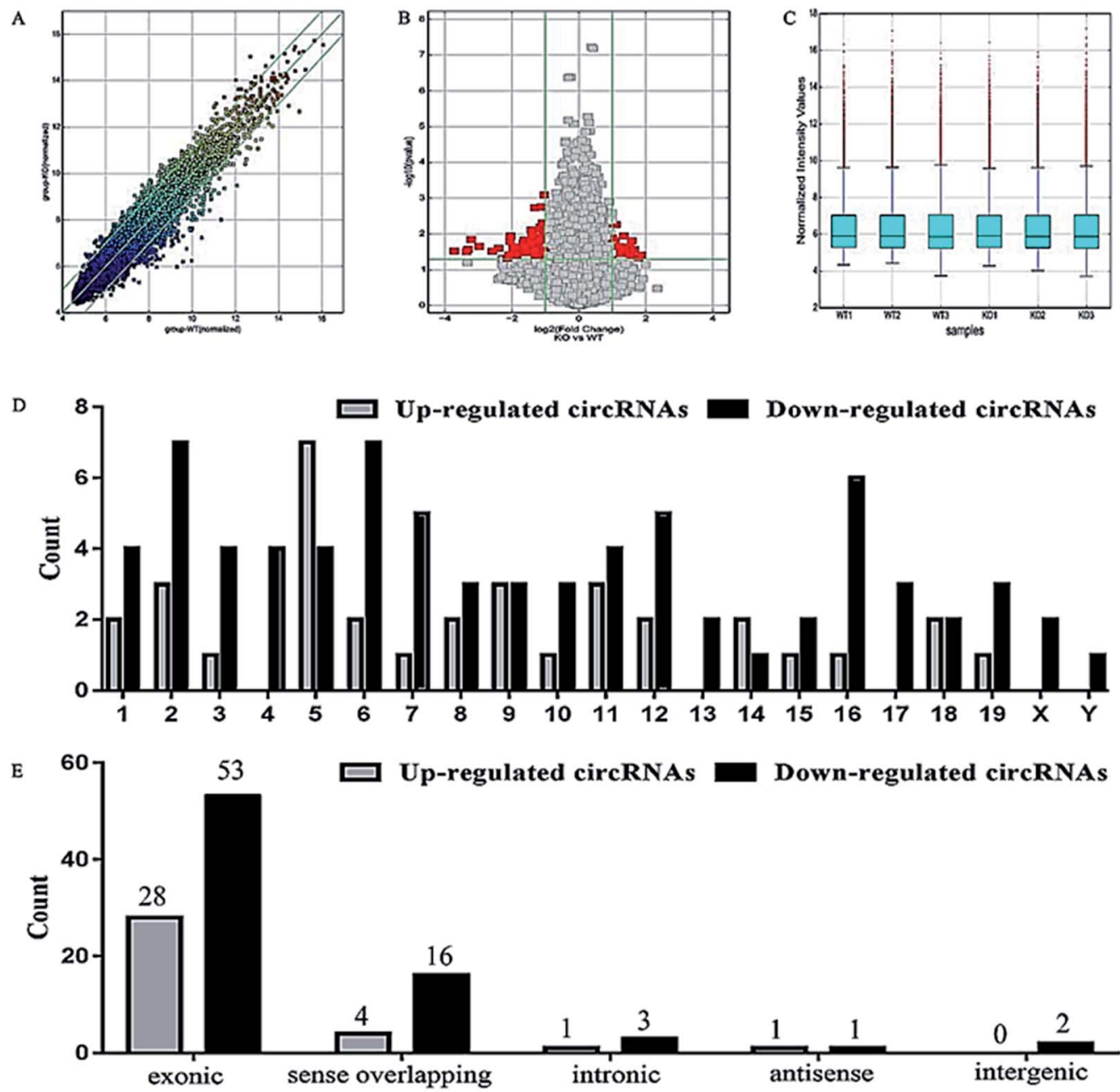

Fig. 3 Screening and analysis of differentially expressed circRNAs in dorsal epidermis from MED1 KO mice and WT mice. (A) Scatter plot showed the variation of circRNAs in expression between MED1 KO mice ( $Y$-axis) and WT mice ( $X$-axis). The values of $x$ and $y$ axes were the normalized signal values (log2 scaled). The green lines are fold change lines. The circRNAs above the top green line and below the bottom green line indicated more than 2.0 fold change of circRNAs between the two compared samples. (B) Volcano plot showed the general distribution of differentially expressed circRNAs. The green vertical lines are the boundary corresponding to 2.0 fold change, and the horizontal line represented $p$-value of 0.05 . The red dots represented the differentially expressed circRNAs in MED1 KO mice vs. WT mice (the left is down-regulation in MED1 KO mice and the right is up-regulation in MED1 KO mice). (C) Box plot showed the homogeneous distributions of the intensities among MED1 KO mice and WT mice. (D) The distribution of differentially expressed circRNAs in mice chromosomes. (E) The histogram showed the classification of differentially expressed circRNAs.

PBS-T (0.1\% Tween 80$)$ for $1 \mathrm{~h}$ followed by primary antibody Tnfrsf19 (bs-3852R, 1 : 200) and Perp (BS91057, $1: 50)$ incubation overnight at $4{ }^{\circ} \mathrm{C}$, slides were rinsed with PBS-T and incubated with secondary antibody (RS0002, $1: 500)$ for $1 \mathrm{~h}$. Color reaction was developed by using 3,3'-diaminobenzidine tetrachloride (DAB) chromogen solution. All slides were counterstained with hematoxylin and followed by examination under a microscope (OLYMPUS).

\section{Statistical analysis}

Statistical analyses were performed using SPSS 21.0 version and Graph pad prism 7.0 version. Data were presented as the mean \pm standard error (SE) for triplicate measurements. The significance of the data was estimated by Student's $t$ test and $P$ value $<0.05$ was considered statistically significant.

\section{Results}

\section{CircRNAs expression profiles in MED1 KO mice}

Here we identified 109 (34-up, 75-down) distinct circRNAs with fold-changes of greater than 2.0 and $p$-value of less than 0.05 from a total of 13304 circRNAs through circRNA microarray that are differently expressed in MED1 KO mice compared with WT mice ( $n=3,10$ weeks old). The hierarchical clustering for all 109 differentially expressed circRNAs was presented in Fig. 2A. The top 15 up-regulated and top 15 down-regulated circRNAs in MED1 KO mice compared with WT mice were listed in ESI 
A
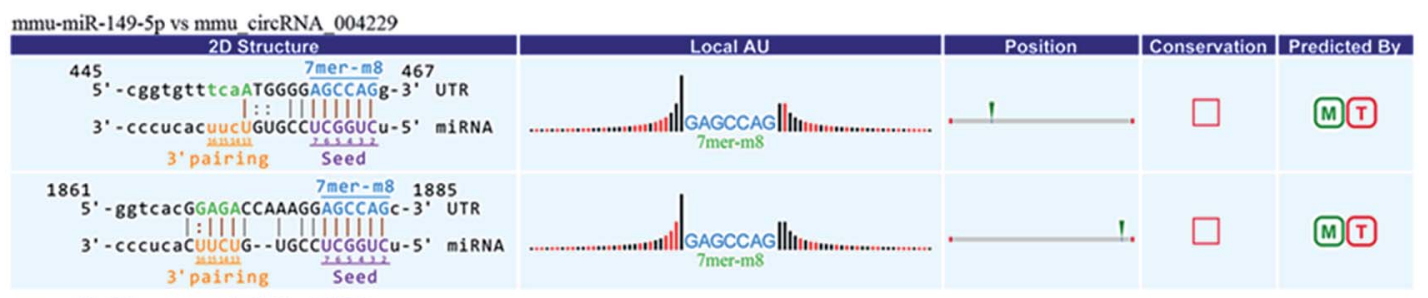

mmu-miR-207 vs mmu_circRNA_004229
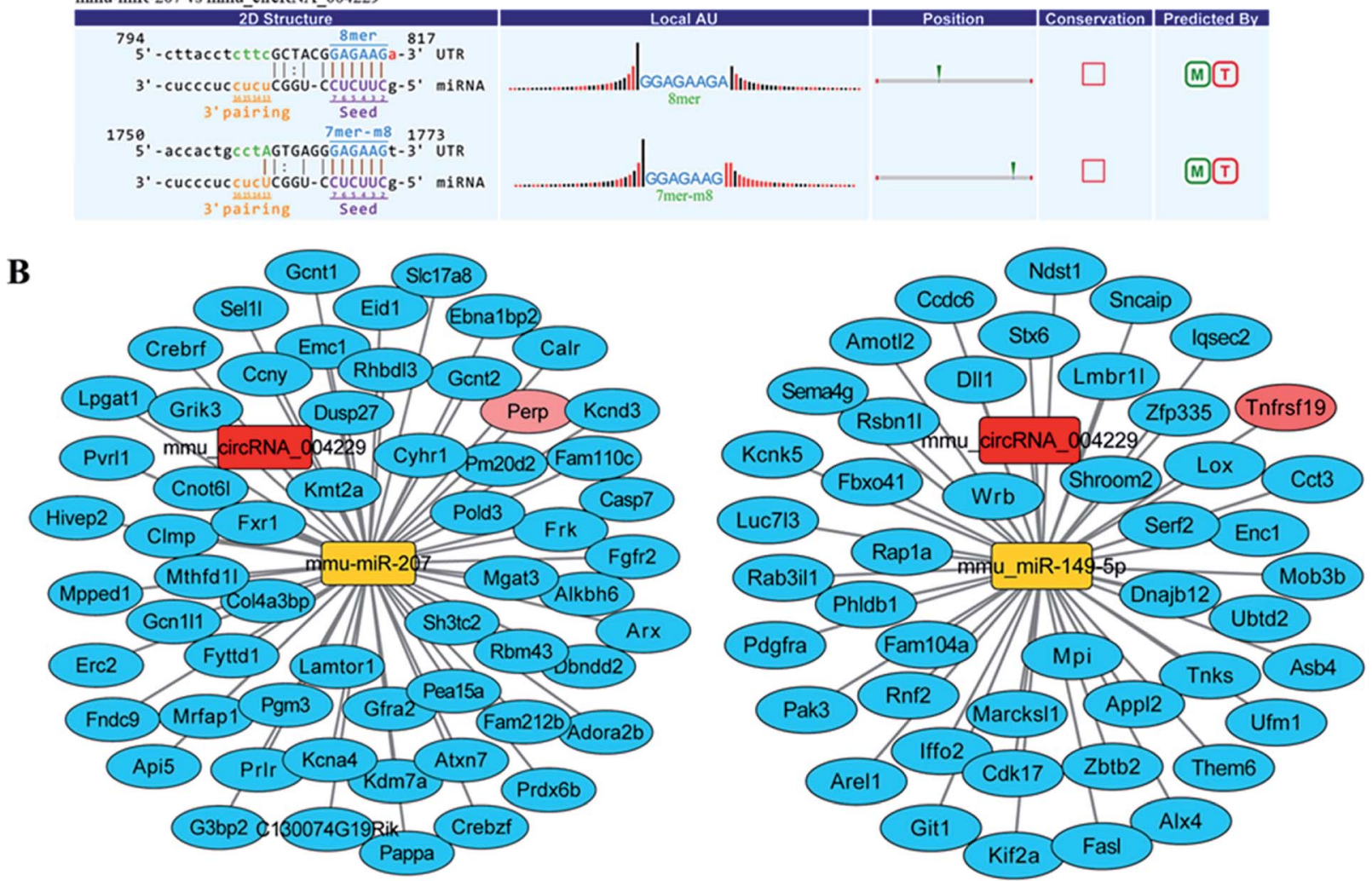

Fig. 4 The biding site prediction and network construction of mmu_circRNA_004229. (A) Binding sites of miR-149-5p/miR-207 in 3'-UTR of mmu_circRNA_004229. (B) Specific network of mmu_circRNA_004229-miR-207/149-5p-targets. We chose targets according to target score $\geq 80$ in miRDB database. We used Cytoscape software 3.5.0 to visualize circRNA_004229-miRNA-targets interactions. In the network, the red rectangle represents circRNA_004229; the yellow rectangle represents miRNA-207/149-5p; the blue oval represents target genes of miRNA207/149-5p and the pink oval represents Perp/Tnfrsf19. The relationship between the nodes was connected with solid lines.

Tables 1 and $2, \uparrow$ and a heat map was drawn to show their expression level (Fig. 2B). The scatter plot and volcano plot showed the variation of circRNA expression between MED1 KO mice and WT mice (Fig. 3A and B), and the box plot showed that the homogeneous distribution of circRNAs for the two compared samples were nearly the same after normalization (Fig. 3C). We did further analysis for the classification of all differentially expressed circRNAs. We found that most were originated from exons. Some were from sense overlapping and intronic, while a few were antisense and intergenic (Fig. 3E). The distribution of all differentially expressed circRNAs on mice chromosomes was demonstrated in Fig. 3D. We confirmed the expression level of differentially expressed circRNAs by qRTPCR. Among the top 15 down-regulated circRNAs, mmu_circRNA_004229 was down-regulated by more than four folds (as the red arrow shows in Fig. 2B). Consistently,
mmu_circRNA_004229 was also observed significantly reduced by 2.3-fold in MED1 KO mice compared to WT mice (Fig. 5A(a)).

\section{Prediction and construction of the circRNA-microRNA-mRNA interaction network}

Recent evidences have demonstrated that circular RNAs play a crucial role in the regulation of gene expression by sequestering the miRNAs. ${ }^{20}$ We used Arraystar's home-made miRNA target prediction software to predict the circRNA/ miRNA interaction and listed the top 5 putative miRNAs of differential circRNAs. We used TargetScan, miRPathDB and miRDB to predict target genes of specific microRNA. The most likely potential target miRNAs for mmu_circRNA_004229 include miR-207, miR-149-5p, miR-7235-3p, miR-667-5p, and miR-6914-5p. The circRNA_004229microRNA-149-5p/microRNA-207-targets networks were 
A a

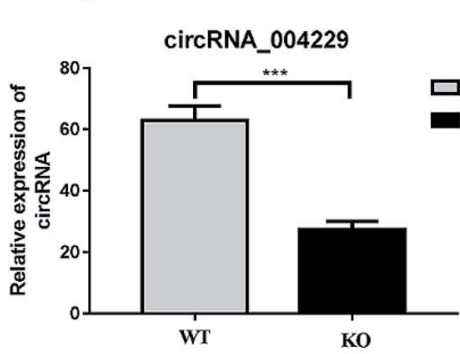

B

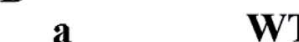

b

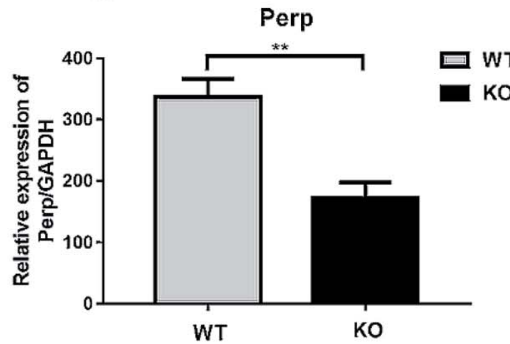

b
KO
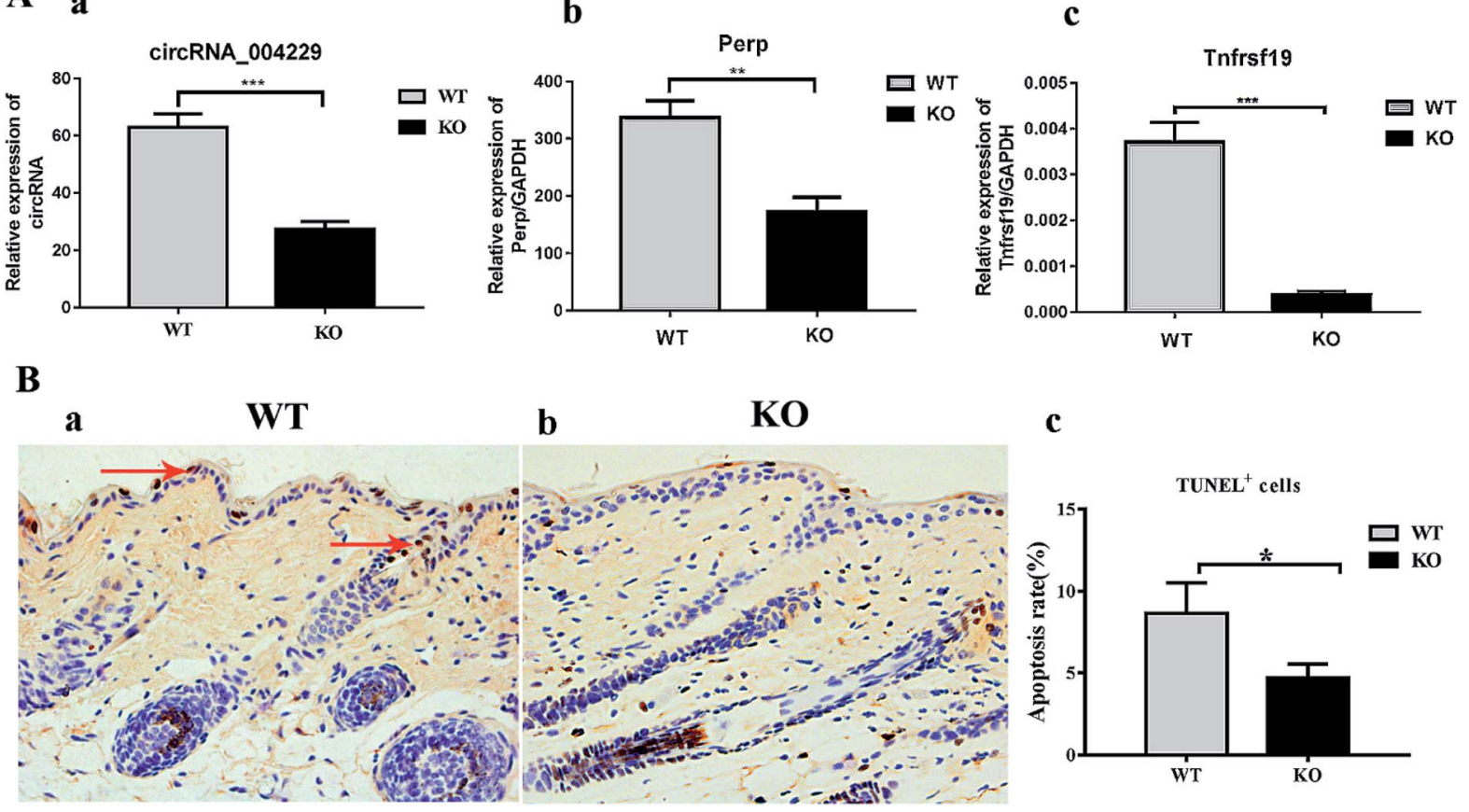

c

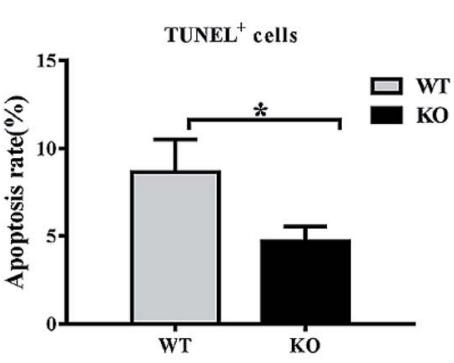

Fig. 5 Mmu_circRNA_004229 was down-regulated and promoted transcriptional inhibition of Perp and Tnfrsf19. (A) QRT-PCR validation for mmu_circRNA_004229 (a) and mRNA levels of Perp (b) and Tnfrsf19 (c) and GAPDH expression was used as a control. (B) TUNEL assay of WT mice (a) and MED1 KO mice (b), the red arrows indicate the apoptosis cells. (c) Quantitative results of TUNEL assay $(n=3, * P<0.05)$.

\section{WT}

KO
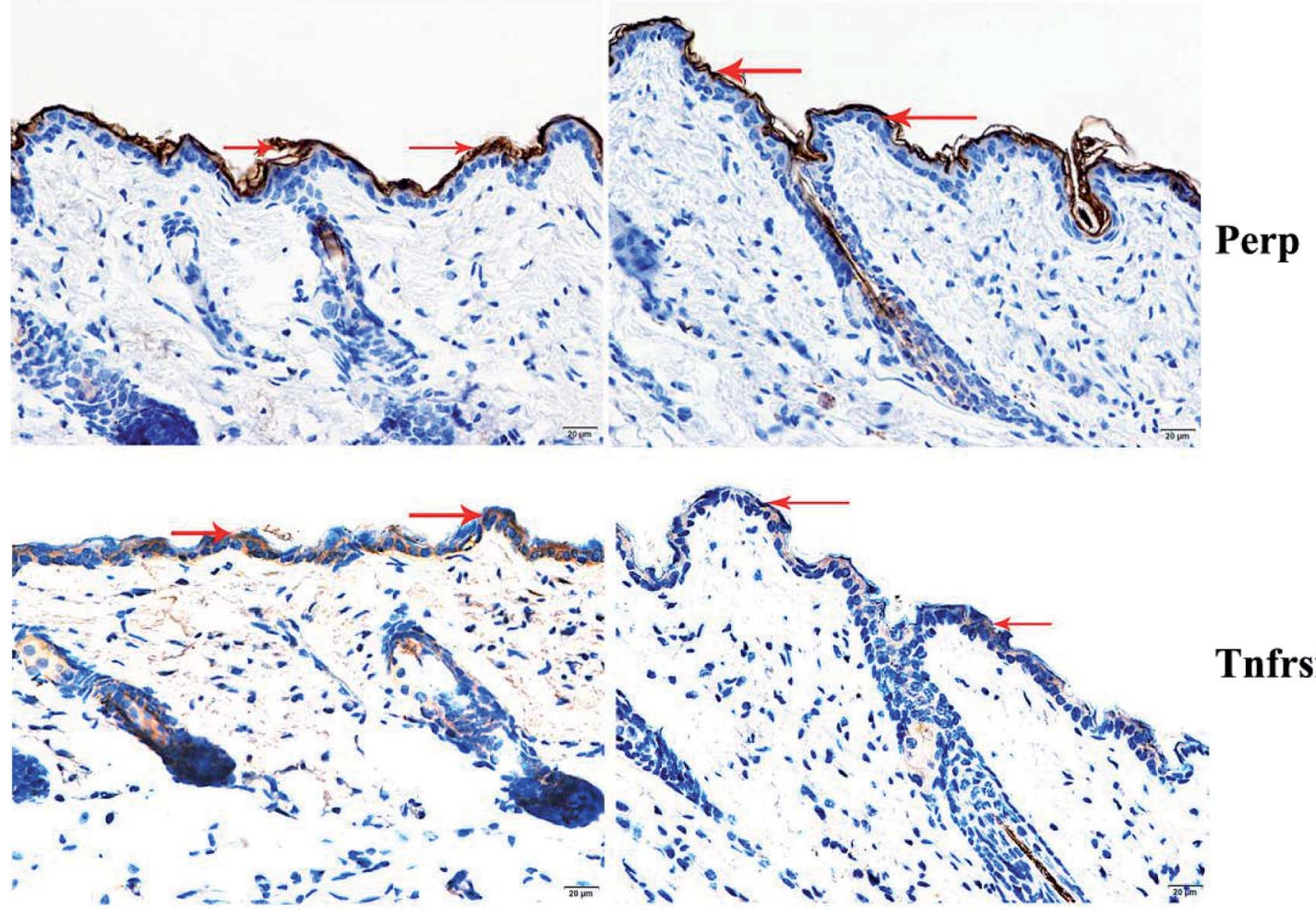

\section{Tnfrsf19}

Fig. 6 Immunohistochemistry results of Perp and Tnfrsf19 and their expression were decreasing slightly in MED1 KO mice (the red arrows indicate). 
constructed to visualize their interactions based on microarray data and microRNA target genes prediction results with Cytoscape 3.5.0 software (Fig. 4B). The results of sequence analysis of microRNA response elements (MRE) for miR-1495p/miR-207 were presented in Fig. 4A. The 2D structure showed the target miRNA seed type (8-mer) and the $3^{\prime}$ pairing sequence (nucleotides 13-16). Two apoptosis-related genes, Tnfrsf19 and Perp, which were potential target genes of miR149-5p and miR-207 respectively, were listed up with high scores.

\section{Reduction of Perp and Tnfrsf19, and suppressed apoptosis in both IFE and HF in MED KO mice}

In order to verify the prediction results obtained, we performed qRT-PCR to validate the Perp and Tnfrsf19 mRNA expression level. Consistent with our expectation, their expression was significantly inhibited in MED1 KO skin compared with control, for 2.0-fold and 10.0-fold down-regulation respectively (Fig. 5A(b and c)). The subsequent immunohistochemical results also showed that Perp and Tnfrsf19 were slightly decreased in MED1 KO epidermis (Fig. 6). Since Perp and Tnfrsf19 were closely related to epidermal development and apoptosis, the apoptosis level was further detected in the dorsal skin of MED1 KO mice and their littermates by TUNEL assay. The apoptosis cells in keratinocytes for both IFE and HF could be observed in WT skin (red arrows, Fig. 5B(a)). However, the number of apoptosis cells were significantly reduced in of MED1 KO keratinocytes (Fig. 5B(b and c)). Thus, the apoptosis was both suppressed in IFE and HF, suggesting a possible process that were under the regulation of MED1 through circRNAs.

\section{Discussion}

Recent studies have demonstrated that circRNAs can function as miRNA 'sponges' in gene regulation for many diseases and biological processes in skin. The circRNAs profiling for clinical skin diseases, such as squamous cell carcinoma, basal cell carcinoma, and severe acne, were reported. ${ }^{15-17}$ CircRNAs also involved in the regulation of pigmentation when comparing the black fur skin and white fur skin. ${ }^{21}$ Moreover, evidence showed that injection of circ-Amotl1 plasmid facilitated wound repair by increasing cell proliferation and migration. ${ }^{18}$ During differentiation of epidermal stem cells, circRNAs profile exhibit a striking change, and upregulated circRNAs might function as miRNA sponges to regulate keratinocyte differentiation. ${ }^{19}$ Therefore, the important role of circRNAs in skin biological function regulation, including the proliferation, differentiation, migration and carcinogenesis, begins to receive a great attention.

MED1, a crucial coactivator for transcription factors in epidermis, is proved to profoundly participate in hair cycling and wound repair regulation. ${ }^{5}$ However, the complex mechanism of MED1 regulating in both HF and IFE is still not fully demonstrated. Thus, exploring the circRNAs change after MED1 deletion in keratinocytes might provide us with some new hints from untouched aspects. In this study, we performed circRNAs microarray to detect the expression profile of circular RNAs. Consistent with our expectation, a great number of circRNAs were identified to upregulate or downregulate after MED1 conditional deletion. In the top decreased circRNAs, we picked mmu_circRNA_004229 as candidate, and confirmed its reduction in MED1 KO epidermis by qRT-PCR. Agnieszka et al. identified mmu_circRNA_004229 was differentially expressed in the brain samples indicating its role in mouse and human brain development. ${ }^{22}$ However, the mechanism of mmu_circRNA_004229 in brain and other tissues needed to be further explored. In this study, mmu_circRNA_004229 might play a role in the regulation of keratinocytes under the control of MED1.

circRNAs could work by possessing ceRNAs activity, binding with miRNAs and strongly reducing the ability for miRNAs to target mRNAs, thus achieving their negative regulation for mRNAs. In this study, we performed the prediction of circRNA/miRNA/ mRNA interaction network to explore the potential function mechanism, especially relating to epidermal biological function. We found that miR-207 and miR-149-5p are potential targets for circRNA_004229. miR-149-5p was reported to suppress cell proliferation and/or promote apoptosis process in melanoma cell via targeting mRNA. ${ }^{23}$ Here, our network prediction indicated Tnfrsf19 (also known as Troy, or Taj), another member in this family, might serve as a potential target for miR-149-5p, which was significantly reduced in MED1 KO skin. Previous research revealed that Tnfrsf19 could bind with TRAF1, TRAF2, TRAF3, and TRAF5 to induce cell death in caspase independent way. ${ }^{24,25}$ Tnfrsf19 was also strongly associated with epidermal development when binding with TRAF6. ${ }^{26-29}$ Together with our results, we assume that MED1 deletion might suppress the function of mmu_circRNA_004229 in sponging miR-149-5p, which in turn promote the binding of miRNA with Tnfrsf19, leading to the regulation of epidermal development regulation, as well as possible suppression of apoptosis for keratinocytes in both HF and IFE.

Further interaction analysis and subsequent expression validation also indicated Perp as target of miR-207. MiR-207 was capable of promoting the apoptosis induced by radiation in cochlea hair cells through targeting Akt3, suggesting the connection of miR-207 with apoptosis process. ${ }^{30}$ Perp, the candidate target of miR-207 in our study, was originally identified as a p53 apoptosis effector. ${ }^{31-34}$ It was reported that overexpression of Perp was able to induce apoptosis. Perp also involved in epithelia maintenance, since Perp null mice died within 10 days after birth for severe blisters in stratified epithelia, including skin and oral mucosa. Further study indicated that Perp achieved its role in skin integrity by influencing the adhesion of keratinocytes after direct activation by $p 63 .^{35}$ Thus, it is possible that reduction of mmu_circRNA_004229/miR-207 in MED1 KO mice results in the suppression of target Perp to carry out its function in apoptosis and/or adhesion of keratinocytes.

Regarding to the limitation of this research, we see present result quite weak in proving our assumption. We only provide circumstantial evidence for possible biological function way of 
mmu_circRNA_004229 by regulating hair/epidermal biological function including apoptosis via miRNA sponging. Further exploration to directly verify those upregulated and downregulated circRNAs function and their underlying mechanisms in regulating epidermal function after MED1 deletion was required for our future study.

\section{Conflicts of interest}

The authors declare no conflict of interest.

\section{Acknowledgements}

This research was supported by the National Natural Science Foundation of China (81573075). We particularly thank the gifts of MED1 KO mice from University of California San Francisco. We thank all author's contributions who participated in this study.

\section{References}

1 F. Barletta, L. P. Freedman and S. Christakos, Enhancement of VDR-mediated transcription by phosphorylation: correlation with increased interaction between the VDR and DRIP205, a subunit of the VDR-interacting protein coactivator complex, Mol. Endocrinol., 2002, 16, 301-314.

2 G. Liu, C. Sprenger, P. J. Wu, S. Sun, T. Uo, K. Haugk, K. S. Epilepsia and S. Plymate, MED1 mediates androgen receptor splice variant induced gene expression in the absence of ligand, Oncotarget, 2015, 6, 288-304.

3 C. Landles, S. Chalk, J. H. Steel, I. Rosewell, B. Spencer-Dene, E. N. Lalani and M. G. Parker, The thyroid hormone receptorassociated protein TRAP220 is required at distinct embryonic stages in placental, cardiac, and hepatic development, Mol. Endocrinol., 2003, 17, 2418-2435.

4 Y. Zhu, C. Qi, S. Jain, M. S. Rao and J. K. Reddy, Isolation and characterization of $\mathrm{PBP}$, a protein that interacts with peroxisome proliferator-activated receptor, J. Biol. Chem., 1997, 272, 25500-25506.

5 Y. Oda, L. Hu, V. Bul, H. Elalieh, J. K. Reddy and D. D. Bikle, Coactivator MED1 ablation in keratinocytes results in haircycling defects and epidermal alterations, $J$. Invest. Dermatol., 2012, 132, 1075-1083.

6 Y. Oda, R. J. Chalkley, A. L. Burlingame and D. D. Bikle, The transcriptional coactivator DRIP/mediator complex is involved in vitamin $\mathrm{D}$ receptor function and regulates keratinocyte proliferation and differentiation, J. Invest. Dermatol., 2010, 130, 2377-2388.

7 X. Q. Zhang and J. H. Yang, Discovering circRNA-microRNA Interactions from CLIP-Seq Data, Methods Mol. Biol., 2018, 1724, 193-207.

8 Y. Yuan, L. Jiaoming, W. Xiang, L. Yanhui, J. Shu, G. Maling and M. Qing, Analyzing the interactions of mRNAs, miRNAs, lncRNAs and circRNAs to predict competing endogenous RNA networks in glioblastoma, J. Neuro-Oncol., 2018, 137, 493-502.
9 B. Chen and S. Huang, Circular RNA: an emerging noncoding RNA as a regulator and biomarker in cancer, Cancer Lett., 2018, 418, 41-50.

10 H. Li, X. Hao, H. Wang, Z. Liu, Y. He, M. Pu, H. Zhang, H. Yu, J. Duan and S. Qu, Circular RNA Expression Profile of Pancreatic Ductal Adenocarcinoma Revealed by Microarray, Cell. Physiol. Biochem., 2016, 40, 1334-1344.

11 L. Chen, S. Zhang, J. Wu, J. Cui, L. Zhong, L. Zeng and S. Ge, circRNA_100290 plays a role in oral cancer by functioning as a sponge of the miR-29 family, Oncogene, 2017, 36, 45514561.

12 X. Wei, H. Li, J. Yang, D. Hao, D. Dong, Y. Huang, X. Lan, M. Plath, C. Lei, F. Lin, Y. Bai and H. Chen, Circular RNA profiling reveals an abundant circLMO7 that regulates myoblasts differentiation and survival by sponging miR378a-3p, Cell Death Dis., 2017, 8, e3153.

13 H. Zhang, G. Wang, C. Ding, P. Liu, R. Wang, W. Ding, D. Tong, D. Wu, C. Li, Q. Wei, X. Zhang, D. Li, P. Liu, H. Cui, H. Tang and F. Ji, Increased circular RNA UBAP2 acts as a sponge of miR-143 to promote osteosarcoma progression, Oncotarget, 2017, 8, 61687-61697.

14 J. Zhang, H. Liu, L. Hou, G. Wang, R. Zhang, Y. Huang, X. Chen and J. Zhu, Circular RNA_LARP4 inhibits cell proliferation and invasion of gastric cancer by sponging miR-424-5p and regulating LATS1 expression, Mol. Cancer, 2017, 16, 151.

15 M. Sand, F. G. Bechara, T. Gambichler, D. Sand, M. Bromba, S. A. Hahn, E. Stockfleth and S. Hessam, Circular RNA expression in cutaneous squamous cell carcinoma, $J$. Dermatol. Sci., 2016, 83, 210-218.

16 M. Sand, F. G. Bechara, D. Sand, T. Gambichler, S. A. Hahn, M. Bromba, E. Stockfleth and S. Hessam, Circular RNA expression in basal cell carcinoma, Epigenomics, 2016, 8, 619-632.

17 J. Liang, X. Wu, S. Sun, P. Chen, X. Liang, J. Wang, J. Ruan, S. Zhang and $\mathrm{X}$. Zhang, Circular RNA expression profile analysis of severe acne by RNA-Seq and bioinformatics, $J$. Eur. Acad. Dermatol. Venereol., 2018, 32, 1986-1992.

18 Z. G. Yang, F. M. Awan, W. W. Du, Y. Zeng, J. Lyu, D. Wu, S. Gupta, W. Yang and B. B. Yang, The Circular RNA Interacts with STAT3, Increasing Its Nuclear Translocation and Wound Repair by Modulating Dnmt3a and miR-17 Function, Mol. Ther., 2017, 25, 2062-2074.

19 L. S. Kristensen, T. L. H. Okholm, M. T. Veno and J. Kjems, Circular RNAs are abundantly expressed and upregulated during human epidermal stem cell differentiation, $R N A$ Biol., 2018, 15, 280-291.

20 S. Ghosal, S. Das, R. Sen, P. Basak and J. Chakrabarti, Circ2Traits: a comprehensive database for circular RNA potentially associated with disease and traits, Front. Genet., 2013, 4, 283.

21 Z. Zhu, Y. Li, W. Liu, J. He, L. Zhang, H. Li, P. Li and L. Lv, Comprehensive circRNA expression profile and construction of circRNA-associated ceRNA network in fur skin, Exp. Dermatol., 2018, 27, 251-257.

22 A. Rybak-Wolf, C. Stottmeister, P. Glazar, M. Jens, N. Pino, S. Giusti, M. Hanan, M. Behm, O. Bartok, R. Ashwal-Fluss, 
M. Herzog, L. Schreyer, P. Papavasileiou, A. Ivanov, M. Ohman, D. Refojo, S. Kadener and N. Rajewsky, Circular RNAs in the Mammalian Brain Are Highly Abundant, Conserved, and Dynamically Expressed, Mol. Cell, 2015, 58, 870-885.

23 W. Chen, J. Zhang, H. Xu, J. Dai and X. Zhang, The negative regulation of miR-149-5p in melanoma cell survival and apoptosis by targeting LRIG2, Am. J. Transl. Res., 2017, 9, 4331-4340.

24 Y. Wang, X. Li, L. Wang, P. Ding, Y. Zhang, W. Han and D. Ma, An alternative form of paraptosis-like cell death, triggered by TAJ/TROY and enhanced by PDCD5 overexpression, J. Cell Sci., 2004, 117, 1525-1532.

25 M. T. Eby, A. Jasmin, A. Kumar, K. Sharma and P. M. Chaudhary, TAJ, a novel member of the tumor necrosis factor receptor family, activates the c-Jun $\mathrm{N}$ terminal kinase pathway and mediates caspaseindependent cell death, J. Biol. Chem., 2000, 275, 1533615342.

26 A. Naito, H. Yoshida, E. Nishioka, M. Satoh, S. Azuma, T. Yamamoto, S. Nishikawa and J. Inoue, TRAF6-deficient mice display hypohidrotic ectodermal dysplasia, Proc. Natl. Acad. Sci. U. S. A., 2002, 99, 8766-8771.

27 S. K. Sinha, S. Zachariah, H. I. Quinones, M. Shindo and P. M. Chaudhary, Role of TRAF3 and -6 in the activation of the NF-kappa B and JNK pathways by X-linked ectodermal dysplasia receptor, J. Biol. Chem., 2002, 277, 44953-44961.

28 J. Pispa, M. Pummila, P. A. Barker, I. Thesleff and M. L. Mikkola, Edar and Troy signalling pathways act redundantly to regulate initiation of hair follicle development, Hum. Mol. Genet., 2008, 17, 3380-3391.
29 T. Kojima, Y. Morikawa, N. G. Copeland, D. J. Gilbert, N. A. Jenkins, E. Senba and T. Kitamura, TROY, a newly identified member of the tumor necrosis factor receptor superfamily, exhibits a homology with Edar and is expressed in embryonic skin and hair follicles, J. Biol. Chem., 2000, 275, 20742-20747.

30 P. X. Tan, S. S. Du, C. Ren, Q. W. Yao, R. Zheng, R. Li and Y. W. Yuan, MicroRNA-207 enhances radiation-induced apoptosis by directly targeting Akt3 in cochlea hair cells, Cell Death Dis., 2014, 5, e1433.

31 M. R. Marques, J. S. Horner, R. A. Ihrie, R. T. Bronson and L. D. Attardi, Mice lacking the p53/p63 target gene Perp are resistant to papilloma development, Cancer Res., 2005, 65, 6551-6556.

32 K. Singaravelu, K. Devalaraja-Narashimha, B. Lastovica and B. J. Padanilam, PERP, a p53 proapoptotic target, mediates apoptotic cell death in renal ischemia, Am. J. Physiol. Renal Physiol., 2009, 296, F847-F858.

33 K. Chen, Z. Luo, Z. Li, Y. Liu and Q. Zhao, PERP gene therapy attenuates lung cancer xenograft via inducing apoptosis and suppressing VEGF, Cancer Biol. Ther., 2011, 12, 1114-1119.

34 R. A. Ihrie, E. Reczek, J. S. Horner, L. Khachatrian, J. Sage, T. Jacks and L. D. Attardi, Perp is a mediator of p53dependent apoptosis in diverse cell types, Curr. Biol., 2013, 13, 1985-1990.

35 R. A. Ihrie, M. R. Marques, B. T. Nguyen, J. S. Horner, C. Papazoglu, R. T. Bronson, A. A. Mills and L. D. Attardi, Perp is a p63-regulated gene essential for epithelial integrity, Cell, 2005, 120, 843-856. 